\title{
Flexibilidad Cognitiva y Comprensión Gramatical en Mayores con Deterioro Cognitivo Leve de Tipo Amnésico
}

\author{
Cognitive Flexibility and Grammatical Comprehension in Older Adults with Mild \\ Cognitive Impairment of the Amnestic Type
}

\author{
Andrea Sánchez-Beato ${ }^{1}$, Marta Galindo ${ }^{2}$, Miriam Gemeno ${ }^{3}$, Alba Jiménez ${ }^{4}$, José María Prados \\ Atienza $^{5}$ y Ramón López-Higes ${ }^{6}$
}

\begin{abstract}
Resumen
Se analiza el papel de la flexibilidad cognitiva en la comprensión de oraciones de mayores con deterioro cognitivo leve de tipo amnésico (DCLa). Participaron 38 pacientes con DCLa, seleccionados a partir de un análisis de conglomerados basado en su ejecución en el Trail Making Test (TMT). Los grupos estaban igualados en educación, edad, estatus cognitivo y memoria, pero diferían en la parte B del TMT, por ello se les denominó: grupo de alta y grupo de baja flexibilidad cognitiva, respectivamente. Resultados: los grupos difieren en las oraciones sintácticamente más simples y de una proposición, específicamente, en los distractores sintácticos. El grupo de baja flexibilidad cognitiva presenta mayor dificultad que el de alta en la comprensión de oraciones simples que requieren un análisis del contenido (distractores sintácticos). Esto indicaría que tienen mayores dificultades para llevar a cabo el reanálisis necesario para formular una respuesta correcta en este tipo de ítems.
\end{abstract}

Palabras clave: deterioro cognitivo leve, análisis de conglomerados, flexibilidad cognitiva, comprensión gramatical, complejidad sintáctica, densidad semántica

\begin{abstract}
The role of cognitive flexibility in the comprehension of sentences of elderly with mild cognitive impairment amnestic type (DCLa) is analyzed. Thirty-eight patients with DCLa participated, selected from a cluster analysis based on their execution in the Trail Making Test (TMT). The groups were matched in education, age, cognitive status and memory, but they differed in part B of the TMT, for that reason they were called: high and low cognitive flexibility groups, respectively. Results: the groups differ in the syntactically simplest sentences and whit one proposition, specifically, in the syntactic foils. The group of low cognitive flexibility presents greater difficulty than the group of high in the comprehension of simple sentences that require an analysis of the content (syntactic foils). This would indicate that they have greater difficulties in carrying out the necessary reanalysis to formulate a correct answer in this type of item.
\end{abstract}

Keywords: mild cognitive impairment, cluster analysis, cognitive flexibility, grammatical comprehension, syntactic complexity, semantic density, syntactic foils

\footnotetext{
${ }^{1}$ Graduada en Logopedia, Máster de trastornos de la comunicación: neurociencia de la audición y el lenguaje, Departamento de Psicología Básica II, Universidad Complutense de Madrid (UCM). Campus de Somosaguas s/n; Pozuelo de Alarcón, 28223 Madrid, España. Tel.: 913943023. Correo: andrsa02@ucm.es

${ }^{2}$ Licenciada en Psicología, Máster en Psicología Clínica. Departamento de Psicología Básica II, UCM. Campus de Somosaguas s/n; Pozuelo de Alarcón, 28223 Madrid, España. Tel.: 913943023. Correo: martagfu@ hotmail.es

${ }^{3}$ Graduada en Psicología, Máster en psicología Clínica. Departamento de Psicología Básica II, UCM. Campus de Somosaguas s/n; Pozuelo de Alarcón, 28223 Madrid, España. Tel.: 913943023. Correo: mgemeno@ gmail.com

${ }^{4}$ Graduada en Logopedia, Máster en intervención logopédica. Departamento de Psicología Básica II, UCM. Campus de Somosaguas s/n; Pozuelo de Alarcón, 28223 Madrid, España. Tel.: 913943023. Correo: albaji03@ucm.es

${ }^{5}$ Doctor en Psicología, Departamento de Psicología Básica II. UCM. Campus de Somosaguas s/n; Pozuelo de Alarcón, 28223 Madrid, España. Tel.: 913943023. Correo: jmpatienza@psi.ucm.es

${ }^{6}$ Doctor en Psicología, Departamento de Psicología Básica II, UCM. Campus de Somosaguas s/n; Pozuelo de Alarcón, 28223 Madrid, España. Tel.: 913943023. Correo: rlopezsa@ psi.ucm.es (Autor para correspondencia)
} 


\section{Introducción}

Una de las principales dificultades que pueden encontrarse a la hora de realizar una evaluación en adultos mayores es llegar a un diagnóstico que permita distinguir entre el envejecimiento normal, entendido como aquel que se caracteriza por ser activo, sano, óptimo, productivo y vital (Danilo, 2010), y el envejecimiento patológico (Ferrante, 2004); dentro de esta última categoría, estaría el deterioro cognitivo leve (DCL). Inicialmente este concepto se centraba en la memoria y su modelo era la Enfermedad de Alzheimer (EA), por ello se diferenciaron tres subtipos que presentaban distinta evolución (Petersen et al., 2001): (a) DCL de tipo amnésico, cuyo principal síntoma es la pérdida de memoria, y si evoluciona a demencia lo hará probablemente a EA; (b) DCL con múltiples funciones alteradas sin que se requiera afectación de la memoria (si evoluciona a demencia puede hacerlo hacia EA, demencia vascular u otros tipos); (c) DCL con una sola función alterada distinta de la memoria, que puede evolucionar hacia otros tipos de demencias (Frontotemporal, Vascular, Cuerpos de Lewy, Afasia primaria progresiva, etc.). Actualmente, cuando se hace un diagnóstico de DCL, se indica el subtipo, por ejemplo "DCL de tipo amnésico", "DCL multidominio con alteraciones en memoria y funciones ejecutivas", etc. (Montenegro, Montejo, Llanero, \& Reinoso, 2012). El DCL se considera un síndrome susceptible de evolución, aunque inicialmente afecte solo a la memoria, también puede tener impacto en la atención, las funciones ejecutivas o el lenguaje a lo largo del tiempo (Ritchie \& Ritchie, 2012; Sperling et al., 2011). En otras ocasiones el síndrome se mantiene estable o incluso puede que las personas con DCL vuelvan a la normalidad (Koepsell \& Monsell, 2012). Las habilidades lingüísticas más estudiadas en pacientes con DCL son la memoria semántica verbal, la fluidez verbal y la denominación (Juncos-Rabadán et al., 2010). Las pocas investigaciones que han estudiado la comprensión oral (o la comprensión gramatical) en estos pacientes han arrojado resultados contradictorios (Hodges et al., 2006; Lambon-Ralph et al., 2003; Ribeiro et al., 2006).

Se sabe que las personas mayores sanas experimentan dificultades con la comprensión oral de las oraciones en un contexto de complejidad sintáctica creciente y demandas relacionadas con la tarea (Wingfield et al., 2003). En un estudio de 241 participantes, con edades comprendidas entre 55 y 88 años (Goral et al., 2011), los autores encontraron que la ejecución en dos tareas de comprensión de oraciones se asociaba con (a) la complejidad de las estructuras oracionales empleadas, (b) la capacidad de la memoria de trabajo y la habilidad para cambiar de conjunto), $\mathrm{y}$, finalmente, con tres variables sociodemográficas: género, educación y edad. Concretamente, en las oraciones de relativo de objeto y con dos partículas negativas, el rendimiento de los participantes fue peor. Los individuos con más capacidad de memoria de trabajo y mayores habilidades de cambio tuvieron un mejor rendimiento en las tareas de comprensión auditiva propuestas. Un hallazgo interesante que los autores subrayan es que las personas con baja capacidad de la memoria de trabajo y poca habilidad para el cambio tuvieron dificultades para comprender oraciones complejas, mientras que otros participantes pudieron compensar la dificultad que entrañaban estos elementos por su mayor capacidad o habilidad.

En un estudio previo (López-Higes et al., 2014), realizado con una muestra de 27 adultos mayores cognitivamente intactos y 19 pacientes con DCL multidominio, se intentó determinar si la comprensión de verbos y de oraciones con diferentes estructuras sintácticas podría depender esencialmente de las capacidades léxicas de los participantes. Para ello se evaluó a los participantes mediante la prueba de denominación del Test de Boston y pruebas de fluidez verbal fonológica y semántica. Los resultados indicaron que los pacientes con DCL multidominio mostraron peor rendimiento en la comprensión de verbos y de oraciones, y que el nivel alcanzado en esas tareas no dependía de sus habilidades léxicas. Sin embargo, el desempeño del grupo de mayores sanos en las tareas de comprensión podría estar relacionado con procesos de control y con problemas de reconocimiento. Adicionalmente, se comprobó que las estructuras oracionales que permitían clasificar (diferenciar) a los dos grupos de participantes no se ajustaban al orden sintáctico canónico del español. 
En otro estudio se comparó el rendimiento de un grupo de pacientes alemanes con enfermedad de Parkinson y un grupo de control en una tarea de verificación oración-dibujo (Colman et al., 2011). Los resultados de este trabajo mostraban que el bajo rendimiento de los pacientes con Parkinson dependía de las funciones ejecutivas y de la flexibilidad cognitiva, evaluadas a través del TMT y del "Odd Man Out test". El peor rendimiento en las oraciones pasivas (no ajustadas al orden sintáctico canónico del alemán) estaba relacionado con una menor flexibilidad cognitiva. Los autores sugirieron que esto podría indicar que la flexibilidad cognitiva es necesaria durante la comprensión de ciertas oraciones (como las pasivas) para deshabilitar una asignación temática (canónica) esperada y aumentar la activación de una asignación no-canónica.

En la vida diaria, las personas mayores usan y comprenden principalmente el lenguaje literal, pero a veces se enfrentan con enunciados no literales que requieren una interpretación bastante exigente de su significado. Estudios recientes han demostrado que, en el continuo de la normalidad a la EA, la comprensión de metáforas comienza a deteriorarse en la fase de DCLa, y en la fase leve de la EA se observa la interpretación literal del lenguaje pragmático (Maki et al., 2013). Por ejemplo, según algunos autores, la Teoría de la Mente y las funciones ejecutivas son requisitos previos para una comprensión de la ironía (Champagne-Lavau \& Joanette, 2009), capacidades que están más deterioradas en individuos con DCL que en personas mayores sanas (Gaudreau et al., 2013).

En un estudio relativamente reciente (Cardoso et al., 2014) los autores informaron de que los pacientes con DCL presentaban puntuaciones más bajas en todas las pruebas complejas de lenguaje, tanto literales como no literales (proverbios, etc.), que el grupo de control. Hasta el 69\% de los participantes con DCL puntuaban 1,5 desviaciones típicas por debajo de la media en al menos una de las pruebas de lenguaje.

De esta revisión surgen ciertas preguntas que pueden formularse como objetivos de investigación. Uno de especial interés se ha planteado también en algunos estudios previos (Hochstadt, 2009; Hochstadt et al., 2006), esto es: si las funciones ejecutivas tienen influencia en la comprensión de oraciones complejas. La comprensión de una oración requiere que el oyente/lector dirija la atención hacia la información lingüística relevante, mantenga la información en la memoria de trabajo durante el desarrollo de la representación del significado e inhiba los análisis incorrectos o las interpretaciones erróneas. En este estudio exploramos los efectos de la flexibilidad cognitiva y la velocidad de procesamiento en la comprensión de oraciones en una muestra de personas mayores con DCL amnésico (DCLa). Como hipótesis general, se sugiere que los pacientes con menor flexibilidad cognitiva y velocidad de procesamiento presentarán un desempeño significativamente peor en la comprensión de oraciones más complejas (con dos proposiciones o dos verbos, o no ajustadas al orden sintáctico canónico del español) que los pacientes con mayor flexibilidad cognitiva y velocidad de procesamiento.

\section{Método}

\section{Participantes}

Se seleccionaron casos de una muestra de 216 adultos mayores (de 63 a 88 años, con o sin DCL), reclutados por los profesionales del Centro de Prevención del Deterioro Cognitivo del Ayuntamiento de Madrid (CPCD), de acuerdo con los siguientes criterios (Petersen et al., 1999; Petersen et al., 2009): (1) Mini Examen Cognitivo (Lobo et al., 1979) mayor o igual a 24 puntos; (2) Recuerdo demorado en Lista de Palabras de la Escala de Memoria de Weschler (WMS-III; adaptación española: Wechsler, 2004) en el percentil 30 o inferior (igual o inferior a dos palabras para sujetos entre 66 y 73 años, o igual a uno o cero para más de 74 años); (3) Presencia de quejas de memoria corroboradas por un informante fiable y un/a profesional del CPCD; (4) Actividades diarias suficientemente bien conservadas: Escala de Evaluación Funcional (Functional Assessment Scale, FAQ) < 6 (Olazarán et al., 2005); (5) No cumple con criterios de demencia.

Todos los participantes firmaron un formulario de consentimiento informado para participar en el estudio. 
Tabla 1. Media y desviación estándar (DS) en las siguientes variables por grupos: MEC, Lista de palabras (WMS- III) recuerdo demorado, edad, errores y tiempo de ejecución en las partes A y B del TMT

\begin{tabular}{lccccc}
\hline & \multicolumn{5}{c}{ Grupos } \\
\cline { 2 - 5 } & \multicolumn{7}{c}{ Alta flexibilidad cognitivaBaja flexibilidad cognitiva } \\
\cline { 2 - 5 } & Media & DS & Media & DS & Sig. diferencia \\
\hline Mini Examen Cognitivo (MEC) & 26.67 & 1.72 & 26.00 & 1.41 & .089 \\
Lista de palabras: recuerdo diferido & .92 & .90 & .60 & .89 & 1.000 \\
Edad & 75.25 & 3.11 & 74.00 & 3.32 & .059 \\
TMT-A errores & .26 & .56 & .016 & .50 & .546 \\
TMT-A tiempo de ejecución & 72.11 & 25.76 & 87 & 39.71 & .179 \\
TMT-B errores & 1.05 & 1.27 & 3.62 & 3.38 & .007 \\
TMT-B tiempo de ejecución & 163.68 & 43 & 247.87 & 49.10 & .000 \\
\hline
\end{tabular}

Aplicando los criterios anteriores, $\mathrm{y}$ descartando la presencia de alteraciones en otros dominios cognitivos distintos de la memoria (funciones ejecutivas o lenguaje) con otros tests que forman parte del protocolo que habitualmente se emplea en el CPDC (Test de Stroop, Golden [1978]; Fluidez verbal fonológica y semántica; Test de denominación de Boston, García-Albea, Sánchez-Bernardos \& del Viso, [1986], o de cuadro depresivo (a través de la Geriatric Depression Scale: GDS-15, Sheikh \& Yesavage [1986]), se constituyó un grupo inicial compuesto por 51 personas que tenían un perfil de DCLa. Se realizó un análisis de conglomerados de $\mathrm{K}$-medias con todos estos casos para establecer dos grupos diferenciados por su desempeño en el TMT (Reitan, 1992), ya que la parte B se considera una medida de flexibilidad cognitiva (Maruta et al., 2011) y la parte A una de velocidad de procesamiento (Lezak et al., 2004). El análisis de conglomerados mostró que los errores y el tiempo de ejecución en la Parte B del TMT fueron las variables que maximizaban las diferencias entre los dos grupos resultantes: $F_{\text {errorsB }}(1,49)=7.18$, $p<.015 ; \quad \mathrm{F}_{\text {timeB }}(1, \quad 49)=97.18, \quad p<.001 . \quad \mathrm{Se}$ descartaron 13 casos que no encajaban bien en ninguno de los grupos resultantes. Los dos grupos finales eran de igual tamaño $\left(\mathrm{n}_{1}=19, \mathrm{n}_{2}=19\right)$ y tenían un nivel educativo semejante; no había diferencias entre grupos ni en el MEC, ni en el recuerdo demorado de la Lista de Palabras del WMS-III, ni en la media de edad. La Tabla 1 muestra las características de los dos grupos.
Los pacientes con DCLa del primer grupo fueron más rápidos y más precisos en la parte $\mathrm{B}$ del TMT (alta flexibilidad cognitiva) que los del segundo grupo (baja flexibilidad cognitiva).

\section{Materiales}

El estado cognitivo de todos los participantes se determinó con el MEC, la Lista de Palabras del WMS-III y la Escala de Evaluación Funcional (FAQ), como se mencionó anteriormente. El Trail Making Test (TMT) se usó como una medida de la flexibilidad cognitiva y de la velocidad de procesamiento.

La comprensión gramatical se evaluó mediante la subprueba de comprensión de oraciones de la prueba ECCO-Senior (Exploración Cognitiva de la Comprensión de Oraciones; López-Higes et al., 2012). Se trata de una prueba de verificación simple (oracióndibujo) en la que se incluyen 12 ítems congruentes y 24 incongruentes (distractores sintácticos y léxicos). En los distractores sintácticos, al comparar el dibujo y la oración los roles temáticos de los participantes están invertidos. En los distractores léxicos, se produce una incongruencia entre el dibujo y la oración con respecto a una acción, un participante o un objeto. Cada tipo de oración tiene características específicas en dos dimensiones: densidad proposicional (número de proposiciones) y ajuste al orden sintáctico canónico del español. En español el orden sintáctico normal (o canónico) es sujeto-verboobjeto (SVO). 


\section{Procedimiento}

Las pruebas neuropsicológicas utilizadas para la selección inicial de los participantes (MEC, FAQ y Lista de Palabras del WSM-III) fueron aplicadas por los profesionales del CPDC en una sola sesión, estableciéndose un orden de presentación aleatorio para cada participante. En otra sesión diferente se administraron el TMT y la prueba de comprensión de oraciones, cuyo orden también variaba de forma aleatoria para cada participante. Todas las pruebas fueron administradas y corregidas de acuerdo con las instrucciones recogidas en sus respectivos manuales.

En la prueba de comprensión de oraciones los participantes tenían que contestar "Verdadero" o "Falso", momento en el que el evaluador presentaba un nuevo elemento. Este procedimiento se repetía hasta el final de la prueba. No se informaba a los participantes si sus respuestas eran correctas o incorrectas durante la realización de la prueba.

\section{Análisis estadístico}

Las pruebas neuropsicológicas se corrigieron $\mathrm{y}$ puntuaron de acuerdo con sus normas de corrección. En la prueba de comprensión de oraciones se obtuvo el total de aciertos en las oraciones ajustadas y no ajustadas al orden sintáctico canónico del español, así como el total en las oraciones de una y de dos proposiciones. Además, se calcularon otras puntuaciones adicionales que tenían en cuenta las dos dimensiones mencionadas (orden sintáctico y número de proposiciones) y el tipo de elemento (congruente, distractor léxico, distractor sintáctico).

Todos los análisis se realizaron con el programa IBM SPSS 20.0. Primero, se realizó el análisis de conglomerados de K-medias. Se utilizó el estadístico $U$ de Mann-Whitney para calcular las diferencias entre los grupos en todas las variables dependientes, ya que es una prueba robusta frente a la violación del supuesto de normalidad. También se realizaron pruebas de Friedman para comprobar si existían diferencias significativas intra-grupo, y de Wilcoxon, para comparaciones de pares post-hoc. Se aplicó la corrección de Bonferroni para ajustar los valores de $p$ en caso de comparaciones múltiples.

\section{Resultados}

La Tabla 2 muestra los estadísticos descriptivos correspondientes a los diferentes índices de la prueba de comprensión de oraciones para los dos grupos de pacientes con DCLa.

\section{Orden canónico y tipo de ítem}

Se encontraron diferencias estadísticamente significativas entre los dos grupos de pacientes en los distractores sintácticos ajustados al orden canónico del español (SVO), Mann-Whitney $U=88 ; Z=-2.84 ; p=.007$.

En el grupo de alta flexibilidad cognitiva, la prueba de Friedman no encontró diferencias significativas entre los tres tipos de ítems en las oraciones ajustadas al orden canónico. Sin embargo, en el grupo de baja flexibilidad cognitiva sí aparecieron diferencias significativas entre los tipos de ítems (Chi-cuadrado (2) = 8.41; $p=.02)$ para este tipo de oraciones. Las comparaciones a posteriori entre los tipos de ítems en oraciones ajustadas al orden canónico (Wilcoxon) con la corrección de Bonferroni ( $p=.017)$ mostraron que el rendimiento en ítems congruentes era significativamente diferente del obtenido en distractores sintácticos $(Z=-2.08$; $p=.04)$ y distractores léxicos $(Z=-2.35 ; p=.015)$.

Al considerar los resultados de los pacientes DCLa con alta flexibilidad cognitiva en las oraciones no ajustadas al orden canónico, se comprobó la existencia de diferencias significativas entre los distintos tipos de ítems, Chi-cuadrado (2)=18.97; $p=.001$. Las diferencias surgen cuando los ítems congruentes se comparan con los distractores sintácticos $(Z=-3.00 ; p=.004)$ y con los distractores léxicos $(Z=-3.25 ; p=.002)$. En el grupo de baja flexibilidad cognitiva también se encontraron diferencias significativas entre tipos de ítems, Chi-cuadrado (2)=17.94; $p=.001$. Las pruebas de Wilcoxon revelaron que esas diferencias significativas aparecen de nuevo cuando se comparan los ítems congruentes con los distractores sintácticos $(\mathrm{Z}=-3.13 ; p=.003)$ y con los distractores léxicos $(Z=-2.71 ; p=.008)$.

\section{Densidad proposicional y tipo de ítem}

Con respecto al número de proposiciones (densidad proposicional) se observaron diferencias 
Tabla 2. Promedio de aciertos y desviación estándar (DS) por grupo en los distintos tipos de oraciones

\begin{tabular}{lcccc}
\hline & \multicolumn{2}{c}{ Grupos } & & \\
& Alta flexibilidad cognitiva & Baja flexibilidad cognitiva \\
\hline & Media & DS & Media & DS \\
O_canónicas & 15.84 & 1.432 & 14.26 & 1.45 \\
O_no_canónicas & 12.00 & 2.03 & 11.21 & 2.09 \\
O_1_proposición & 14.79 & 1.35 & 13.47 & 1.80 \\
O_2_proposiciones & 13.05 & 1.98 & 12.00 & 1.79 \\
OC_congruentes & & & 5.37 & .76 \\
OC_distractores sintácticos & 5.58 & .61 & 4.52 & .90 \\
OC_distractores léxicos & 5.37 & .89 & 4.37 & 1.16 \\
OnoC_congruentes & 4.89 & 1.15 & 5.00 & 1.41 \\
OnoC_distractores sintácticos & 5.00 & .94 & 3.42 & .90 \\
OnoC_distractores léxicos & 3.79 & 1.03 & 2.79 & 1.58 \\
O1P_congruentes & 3.21 & 1.18 & 5.31 & .88 \\
O1P_distractores sintácticos & 5.16 & .89 & 4.63 & .76 \\
O1P_distractores léxicos & 5.32 & .67 & 3.52 & 1.26 \\
O2P_congruentes & 4.31 & .67 & 5.05 & 1.17 \\
O2P_distractores sintácticos & 5.42 & .84 & 3.31 & 1.06 \\
O2P_distractores léxicos & 3.84 & 1.26 & 3.63 & 1.57
\end{tabular}

Nota: Oraciones ajustadas al orden canónico del español y no ajustadas, oraciones con una proposición y con dos proposiciones. OC: oraciones ajustadas al orden sintáctico canónico; OnoC: oraciones no ajustadas al orden canónico; O1P: oraciones de una proposición; $\mathrm{O} 2 \mathrm{P}$ : oraciones de dos proposiciones

significativas entre los dos grupos de pacientes en distractores sintácticos que incluyen solo una proposición (o verbo), Mann-Whitney $U=97 ; Z=-$ $2.70 ; p=.014$.

En el grupo de pacientes con alta flexibilidad cognitiva la prueba de Friedman reveló diferencias significativas entre los tipos de ítems en oraciones que incluyen solo una proposición, Chi-cuadrado (2)=12.04; $p=.003$. Las comparaciones entre tipos de ítems que resultaron significativas para esta categoría de oraciones fueron las de los distractores léxicos, bien con los ítems congruentes $(Z=-2.60 ; p=.01)$, o bien con los distractores sintácticos $(Z=-3.04 ; p=.003)$. En el grupo de baja flexibilidad cognitiva, la prueba de Friedman reveló también la existencia de diferencias entre tipos de ítems en oraciones con una única proposición, Chi-cuadrado (2)=16.67; $p=.001$. En este caso, los pacientes presentaban resultados significativamente mejores en ítems congruentes que en distractores léxicos, $Z=-3.23$; $p=.002$, y en los distractores sintácticos en comparación con los distractores léxicos, $Z=-2.87$; $p=.005$.

Con respecto a las oraciones que incluyen dos proposiciones, se encontraron diferencias significativas debidas al tipo de ítem en el grupo de pacientes con alta flexibilidad cognitiva, Chicuadrado $(2)=8.09 ; p=.02$. Las comparaciones de pares revelaron la existencia de diferencias significativas entre los elementos congruentes y el resto de ítems: con los distractores sintácticos $(Z=-2.98 ; p=.004), \mathrm{y}$ con los distractores léxicos $(Z=-2.95 ; p=.004)$. En el grupo de pacientes DCLa con baja flexibilidad cognitiva también se observaron diferencias debidas al tipo de ítem en las oraciones de dos proposiciones, Chi-cuadrado $(2)=9.72 ; p=.009 ;$ y la única comparación que alcanzó significación estadística (de acuerdo con la corrección de Bonferroni) fue la que involucraba elementos congruentes y distractores sintácticos $(Z=-2.98 ; p=.004)$.

\section{Discusión}

Los resultados indican que cuando aumentan las demandas (debido a la complejidad sintáctica o a la mayor densidad proposicional), los dos grupos de pacientes con DCLa (con alta y baja flexibilidad cognitiva) alcanzan niveles semejantes en comprensión de oraciones. Este resultado estaría en contra de la hipótesis general que establecíamos al final de la introducción. 
Como también han mostrado estudios previos con diferentes poblaciones, las oraciones que siguen el orden canónico se comprenden mejor que las oraciones no ajustadas al mismo. Además, las oraciones que incluyen una proposición son más fáciles de comprender que las que tienen dos (Diéguez-Vide \& Peña-Casanova, 2012; Wingfield et al., 2006). Aunque en general los pacientes DCLa de baja flexibilidad cognitiva mostraron peor rendimiento en todos los índices relacionados con la comprensión gramatical, no hubo diferencias sustanciales con el otro grupo (de alta flexibilidad cognitiva) en cuanto a la comprensión de las oraciones más exigentes y complejas en términos sintácticos (oraciones no ajustadas al orden canónico), o semánticos (oraciones con dos proposiciones).

Al considerar las oraciones más simples en términos sintácticos y las que tienen una sola proposición, sí aparecieron diferencias significativas entre los dos grupos de pacientes. Los pacientes DCLa con alta flexibilidad cognitiva mostraban mejor comprensión de estas oraciones que los pacientes con baja flexibilidad cognitiva. La causa de esta diferencia entre los grupos no puede radicar en el proceso de "mapping" temático (quién hizo qué a quién), dado que incluso los pacientes afásicos que muestran una comprensión asintáctica son capaces de realizar esta operación, empleando para ello una estrategia de asignación temática simple basada en el orden lineal de los constituyentes (para una discusión en profundidad sobre este punto, ver: Del Río \& López-Higes, 2003; Diéguez-Vide \& Peña-Casanova 2012). Por lo tanto, el origen de esta diferencia debe establecerse en la etapa de verificación, cuando se comparan el significado de la oración y el dibujo (Meltzer \& Braun, 2011). Los resultados obtenidos en el estudio ponen de manifiesto la importancia de los distractores sintácticos más simples (tanto en términos sintácticos como semánticos). En este tipo de elementos, al producirse una incongruencia entre la oración y el dibujo (los roles temáticos se encuentran invertidos en el dibujo con respecto a lo que establece la oración), se requiere un análisis del contenido proposicional para dar la respuesta correcta (falso).
Otro resultado que merece un comentario sería el bajo rendimiento de los participantes ante distractores léxicos no ajustados al orden canónico del español, ya que en esta condición aquél se acerca al nivel de acierto por azar (3/6). La detección de la incongruencia en distractores léxicos se puede realizar de una manera más superficial (Waters, Rochon, \& Caplan, 1998), por tanto este resultado podría indicar que cuando la comprensión es más demandante, los pacientes DCLa -en general- no detectaban en la imagen algo que no aparecía mencionado en la oración que leyeron previamente. Esto sería un signo de limitación atencional general o una mayor susceptibilidad a la distracción (McGinnis, 2012). Los distractores léxicos de una sola proposición fueron los elementos más difíciles de comprender para todos los pacientes, de manera que el patrón general mencionado anteriormente también se confirma para una condición particular.

Con respecto al resto de las comparaciones, se ha comprobado que los pacientes con alta flexibilidad cognitiva tuvieron mejor rendimiento en las condiciones más exigentes en los ítems congruentes que en los distractores, pero no se encontraron diferencias entre distractores léxicos y sintácticos. Este patrón es similar al observado para el grupo de pacientes con baja flexibilidad cognitiva en las condiciones más demandantes y ante las oraciones que siguen el orden canónico, aunque la comparación entre los elementos congruentes y los distractores léxicos que incluyen dos proposiciones solo se aproximó al nivel de la significación estadística tras la corrección de Bonferroni.

Los déficits ejecutivos son comunes en individuos con DCLa (Belanger et al., 2010; Belleville et al., 2008), lo que probablemente explica la ausencia de diferencias entre los dos grupos en las oraciones más demandantes. Como sugieren Colman et al. (2011), este resultado podría implicar que durante la comprensión de oraciones complejas (como las construcciones pasivas y las oraciones de relativo de objeto) la flexibilidad cognitiva es necesaria para deshabilitar una asignación temática canónica esperada y aumentar la activación de una asignación no canónica. Sin embargo, nuestros resultados permiten concluir que los pacientes DCLa con baja flexibilidad cognitiva también 
muestran un nivel de comprensión más bajo en los distractores sintácticos simples que los pacientes con DCLa con alta flexibilidad cognitiva. Una posible explicación de este hecho podría basarse en algunos estudios, como el de Meltzer y Braun (2011), en los que la dificultad asociada con un distractor sintáctico en una tarea de verificación se interpreta como reflejo de los procesos de reanálisis necesarios para responder a ese tipo de ítem correctamente. Cuando los sujetos leen la oración correspondiente a un distractor sintáctico, hacen la asignación de los roles temáticos a los constituyentes de la oración para desarrollar su representación del significado. Luego, la inspección del dibujo provocaría un reanálisis (consciente y controlado) de esta asignación previa, cuando se comprueba que los roles temáticos se han invertido. El reanálisis se ha relacionado con el control atencional (Kane et al., 2007), por lo que la flexibilidad cognitiva es un plausible factor condicionante en este caso (ver Tirapu-Ustárroz et al., 2008a y b para una revisión centrada en modelos de control ejecutivo). Si esto es cierto, lo que hemos observado en nuestro estudio es que los pacientes con DCLa de baja flexibilidad cognitiva muestran mayor resistencia al reanálisis en el caso de los distractores sintácticos.

En conclusión, podríamos decir que todos los pacientes DCLa del estudio tienen problemas para comprender oraciones complejas, es decir, oraciones que no se ajustan al orden canónico (pasivas, etc.) o que incluyen dos proposiciones. Todos los pacientes han mostrado también signos de distracción o déficit atencional. Los participantes con baja flexibilidad cognitiva tienen mayores dificultades que otros participantes con alta flexibilidad a la hora de comprender distractores sintácticos simples. Este resultado probablemente significa que no son suficientemente flexibles para realizar el reanálisis requerido en este tipo de elementos. Si consideramos que la información escrita combinada con imágenes es utilizada con frecuencia por los pacientes con DCLa para razonar y para hacer juicios o tomar decisiones, es importante tener en cuenta que la falta de flexibilidad cognitiva podría estar asociada con una tendencia a mantener la primera idea o elección. La mejora de estos problemas debería ser un objetivo de la intervención y formar parte de una estrategia de rehabilitación de la comprensión del lenguaje (ver, por ejemplo, Eggenberger et al., 2013).

Aunque el estudio presente se ha centrado en la comparación de dos pequeños conglomerados de pacientes con DCLa tiene la ventaja de que los grupos están igualados en diferentes variables (edad, estado cognitivo general, memoria episódica, nivel educativo) y solo difieren en su flexibilidad cognitiva. Por lo tanto, se puede afirmar con un alto grado de confianza que las diferencias encontradas en comprensión gramatical dependerán en gran medida del rasgo que diferencia a los grupos. Para confirmar el patrón de resultados obtenido aquí sería recomendable realizar una investigación futura con un mayor número de pacientes. Una línea de desarrollo potencial de este trabajo podría explorar el curso del procesamiento durante la tarea de verificación en adultos mayores cognitivamente intactos y en pacientes con DCL.

\section{Referencias}

Belanger, S., Belleville, S., \& Gauthier, S. (2010). Inhibition impairments in Alzheimer's disease, mild cognitive impairment and healthy aging: Effect of congruency proportion in a Stroop task. Neuropsychologia, 48, 581-589. doi:10.1016/j.neuropsychologia.2009.10.021

Belleville, S., Bherer, L., Lepage, E., Chertkow, H., \& Gauthier, S. (2008). Task switching capacities in persons with Alzheimer's disease and mild cognitive impairment. Neuropsychologia, 46(8), 2225-2233. doi:10.1016/j.neuropsychologia.2008.02.012

Cardoso, S., Silva, D., Maroco, J., de Mendonça, A., \& Guerreiro, M. (2014). Non-literal language deficits in mild cognitive impairment. Psychogeriatrics, 14, 222-228. doi:10.1111/psyg.12101

Champagne-Lavau, M., \& Joanette, Y. (2009), Pragmatics, theory of mind and executive functions after a right-hemisphere lesion: Different patterns of deficits. Journal of Neurolinguistics, 22(5). 413426. doi:10.1016/j.jneuroling.2009.02.002 
Colman, K. S. F. Koerts, J. Stowe, L. A. Leenders, K. L., \& Bastiaanse, R. (2011). Sentence comprehension and its association with executive functions in patients with Parkinson's Disease. Parkinson's Disease, 115. doi:10.4061/2011/213983

Danilo, R. (2010). Envejecimiento activo. Contribuciones de la psicología. Revista iberoamericana de diagnóstico y evaluación psicológica-E Avaliação Psicológica, 30(2), 163-165.

Del Río, D., \& López-Higes, R. (2003) Especificidad del área de Broca en la comprensión de oraciones. Revista de Logopedia, Foniatría y Audiología, 23, 154163.

Diéguez-Vide, F. \& Peña-Casanova, J. (2012). Comprensión oral (audición) y escrita (lectura). In F. Diéguez-Vide and J. PeñaCasanova, Cerebro y lenguaje: Sintomatología neurolingüística (pp. 209-260) Madrid: Panamericana.

Eggenberger, E., Heimerl, C., \& Bennett, M.I. (2013). Communication skills training in dementia care: A systematic review of effectiveness, training content, and didactic methods in different care settings. International Psychogeriatrics, 25(3), 345358. doi:10.1017/S1041610212001664

Ferrante, V. (2004). Indicadores cognitivos y perceptivos-motores de envejecimiento. Revista Iberoamericana de Diagnóstico y Evaluación - e Avaliação Psicológica. 18(2), 137-159.

García-Albea, J. E., Sánchez-Bernardos, M. L., \& del Viso, S. (1986). Test de Boston para el diagnóstico de la afasia: Adaptación española. En H. Goodglass and E. Kaplan (Eds.): La evaluación de la afasia y de trastornos relacionados. Madrid: Editorial Medica Panamericana, pp 129-198.

Gaudreau, G., Monetta, L., Macoir, J., Laforce, R. Jr., Poulin, S., et al. (2013). Verbal irony comprehension in older adults with amnestic mild cognitive impairment. Neuropsychology, 27(6), 702-712. doi:10.1037/a0034655

Golden, C. J. (1978). Stroop color and word test: Manual for clinical and experimental uses. Chicago, IL: Stoelting.
Goral, M. Clark-Cotton, M. Spiro, A. Obler, L.K. Verkuilen, J., \& Albert, M.L. (2011). The contribution of set switching and working memory to sentence processing in older adults. Experimental Aging Research, 37(5), 516-538. doi:10.1080/0361073X.2011.619858

Hochstadt, J. (2009). Set-shifting and the on-line processing of relative clauses in Parkinson's disease: Results from a novel eye-tracking method. Cortex, 45, 991-1011. doi:10.1016/j.cortex.2009.03.010

Hochstadt, J. Nakano, H. Lieberman, P., \& Friedman, J. (2006). The roles of sequencing and verbal working memory in sentence comprehension deficits in Parkinson's disease. Brain and Language, 97, 243-257. doi:10.1016/j.bandl.2005.10.011

Hodges, J. R., Erzinclioglu, S., \& Patterson, K. (2006). Evolution of cognitive deficits and conversion to dementia in patients with mild cognitive impairment: A very-long-term follow-up study. Dementia and Geriatric Cognitive Disorders, 21, 380-391. doi:10.1159/000092534

Juncos-Rabadán, O. Pereiro, A.X. Facal. D., \& Rodríguez, N. (2010). Una revisión de la investigación sobre lenguaje en el deterioro cognitivo leve. Revista de Logopedia, Foniatría y Audiología, 30, 78-83. doi:10.1016/S0214-4603(10)70119-4

Kane, M. J., Conway, A. R. A., Hambrick, D. Z., \& Engle, R. W. (2007). Variation in working memory capacity as variation in executive attention and control. In A.R.A. Conway, C. Jarrold, M.J. Kane, A. Miyake and J.N. Towse, Variation in Working Memory. (pp. 21-48) New York: Oxford University Press.

Koepsell, T. D. \& Monsell, S. E. (2012). Reversion from mild cognitive impairment to normal or near-normal cognition: Risk factors and prognosis. Neurology, 79,1591-1598. doi: 10.1212/WNL.0b013e31826e26b7

Lezak, M. D. Howieson, D. B., \& Loring, D. W. (2004). Orientation and attention. In M.D Lezak, D.B. Howieson and D.W. Loring, $\left(4^{\mathrm{a}}\right.$ ed) Neuropsychological assessment. (pp. 337374) Oxford, UK: Oxford University Press.

Lobo, A. Ezquerra, J. Gómez-Burgada, F. Sala, J. M., \& Seva-Díaz, A. (1979). El mini-examen cognoscitivo, un test sencillo, practico, para 
detectar alteraciones intelectuales en pacientes médicos. Actas Luso Esp Neurol Psiquiatr Cienc Afines, 7, 189-202.

Lambon-Ralph, M. A. Patterson, K. Graham, N. Dawson, K., \& Hodges. J. R. (2003). Homogeneity and heterogeneity in mild cognitive impairment and Alzheimer's disease: A cross-sectional and longitudinal study of 55 cases. Brain, 126, 2350-2362. doi:10.1093/brain/awg236

López-Higes, R. Prados, J. M. Montejo, P. Montenegro, M., \& Lozano, M. (2014). Is there a grammatical comprehension deficit in multidomain mild cognitive impairment? Universitas Psychologica, 13(4), 1569-1579. doi:10.11144/Javeriana.UPSY13-4.iged

López-Higes, R. Rubio, S. Martín, M.T. Del Río, D., \& Mejuto, G. (2012). Evaluación de la comprensión gramatical en el envejecimiento normal y patológico: Un resumen de los resultados obtenidos con las baterías ECCO y ECCO_Senior. Int J Psychol Res, 5, 96-108.

Maki, Y., Yamaguchi, T., Koeda, T., \& Haruyasu, Y. (2013). Communicative competence in Alzheimer's disease. Metaphor and sarcasm comprehension. Am J Alzheimer Dis Other Demen, 28(1), 69-74. doi:10.1177/1533317512467677

Maruta, C. Guerreiro, M. de Mendonça, A. Hort, J., \& Scheltens, P. (2011). The use of neuropsychological tests across Europe: The need for a consensus in the use of assessment tools for dementia. European Journal of Neurology, 18(2), 279-285. doi:10.1111/j.1468-1331.2010.03134.x

McGinnis, D. (2012). Susceptibility to distraction during reading in young, young-old, and oldold adults. Experimental Aging Research, 38, 370-393 doi:10.1080/0361073X.2012.699365

Meltzer, J., \& Braun, A. (2011). An EEG-MEG dissociation between online syntactic comprehension and post hoc reanalysis. Frontiers in Human Neuroscience, 4, 5-10. doi:10.3389/fnhum.2011.00010

Montenegro, M., Montejo, P., Llanero, M., \& Reinoso, A. I. (2012). Evaluación y diagnóstico del deterioro cognitivo leve. Revista de Logopedia, Foniatría y Audiología, 32(2), 47-56. doi.org/10.1016/j.rlfa.2012.03.002

Olazarán, J., Mouronte, P., \& Bermejo, F. (2005). Validez clínica de dos escalas instrumentales en la enfermedad de Alzheimer. Neurología, 20, 395-401. doi:10.1016/j.ft.2009.12.0

Petersen, R. C., Dolly, R., Kurtz, A., Mohs, R. C., Morris, J. C.... Winblad, B. (2001). Current concepts in mild cognitive impaiment. Archive of Neurology, 58, 1985-1992.

Petersen, R. C., Smith G. E., Waring S.C., Ivanik, R. J., Tangelos, E., \& Kokmen, E. (1999). Mild cognitive impairment: Clinical characterization and outcome. Arch Neurol, 56(3), 303-308.

Reitan, R. M. (1992). Trail Making Test: Manual for administration and scoring. Reitan Neuropsychology Laboratory, Tucson, AZ. doi:10.1016/S0887-6177(03)00042-8

Ribeiro, F. de Mendoza, A., \& Guerreiro, M. (2006). Mild cognitive impairment: Deficits in cognitive domains other than memory. Dementia and Geriatric Cognitive Disorders, 21 (5-6), 284-90. doi: 10.1080/13803390600629775

Ritchie, K., \& Ritchie, C. W. (2012). Mild cognitive impairment (MCI) twenty years on. International Psychogeriatrics, 24, 1-5. doi:10.1017/S1041610211002067.

Sheikh, J. I., \& Yesavage, J. A. (1986). Geriatric Depression Scale (GDS). Recent evidence and development of a shorter version. In T. L. Brink (Ed.), Clinical gerontology: A guide to assessment and intervention (pp. 165-173). New York, NY: The Haworth Press.

Sperling, R.A. et al. (2011). Toward defining the preclinical stages of Alzheimer's disease: Recommendations from the National Institute of Aging-Alzheimer's Association workgroups on diagnostic guidelines for Alzheimer's disease. Alzheimer Dementia, 7, 280-292. doi:10.1016/j.jalz.2011.03.003

Tirapu-Ustárroz, J., García-Molina, A., LunaLario, P., Roig-Rovira, T., \& Pelegrín-Valero, C. (2008a). Modelos de funciones y control ejecutivo (I). Rev. Neurol., 46(11), 684-692.

Tirapu-Ustárroz, J., García-Molina, A., LunaLario, P., Roig-Rovira, T., \& Pelegrín-Valero, C. (2008b). Modelos de funciones y control ejecutivo (II). Rev. Neurol. 46(12), 742-750.

Waters, G. S. Rochon, E., \& Caplan, D. (1998). Task demands and sentence comprehension in patients with dementia of the Alzheimer's Type. Brain and Language, 62, 361-397. doi:10.1006/brln.1997.1880 
Wechsler, D. (2004). WMS-III. Escala de memoria de Wechsler-III: manual de aplicación y puntuación. Madrid: TEA Ediciones.

Wingfield, A. McCoy, S. L. Peelle, J. E. Tun, P. A., \& Cox, L.C. (2006). Effects of adult aging and hearing loss on comprehension of rapid speech varying in syntactic complexity. $J \mathrm{Am}$ Acad Audiol, 17, 487-497. doi:10.3766/jaaa.17.7.4

Wingfield, A. Peelle, J. E., \& Grossman, M. (2003). Speech rate and syntactic complexity as multiplicative factors in speech comprehension by young and older adults. Aging, Neuropsychology, and Cognition: A Journal on Normal and Dysfunctional Development, $\quad 10, \quad 310-322$. doi:10.1076/anec.10.4.310.28974 